\title{
Teaching Reforms in Automotive Power train and Chassis Control System Experiment
}

\author{
Luo Xiao ${ }^{1}$, Wu Jianzhen ${ }^{2}$, Chen Xiaolong ${ }^{3}$ \\ College of Electronic and Electrical Engineering, Shanghai University of Engineering Science, Shanghai ${ }^{1,2,3}$
}

\begin{abstract}
The Automotive Power train and Chassis Control System Experiment is a supporting course for Automotive Power train Control System and Chassis Control System. This paper discusses the choice of experimental materials and how to vividly explain theoretical knowledge and conduct experiments.
\end{abstract}

Keywords: Power train Control System, Chassis Control System, Experimental Methods, Reform

\section{INTRODUCTION}

'Automotive Power train and Chassis Control System Experiment is a comprehensive experimental course of the automotive electronics engineering of college of electronic and electrical engineering. The course is the supporting experiment course of 'Automobile engine control system' ,'Chassis systems control', 'Automobile body control system' and 'Vehicle cruise control system'. The automobile electronic control system mainly includes the electronic control fuel injection system, the electric control ignition system, idle speed control system, automatic transmission, Antilock brake control system, driving anti slip control system, electronic control suspension system, CAN bus control system, cruise control system and safety air bag control system. There are too many experiments but the experiment takes only 3 weeks. It is the question which the experiment arrangement should fully consider about how to make use of the limited experiment time and to let the students master the knowledge which is studied. In the course of teaching methods and experimental reform, the following aspects can be focused on:

\section{MAKES FULL USE OF ENGINE MEASUREMENT AND CONTROL EXPERIMENTAL PLATFORMS}

Our automotive electronics laboratory has a number of engine measurements and control laboratories, which can do the experiments of fuel injection experiments, ignition control system experiments and idle speed control system. Using these experimental platforms, students can master knowledge of the censors, controllers and actuators of these control systems, thus deepening understanding of theoretical knowledge.

1) Intake System: The students need to point out the installation location of the air flow meter, the manifold absolute pressure sensor, the air inlet temperature sensor, the throttle body, and identify the corresponding diagram of control circuit, observe the number of each wire harness and it's thickness, color, etc. Then students also need to contrast the circuit of each sensor actuator in the schematic diagram of the control system, and remember the connection relationship between them and ECU.
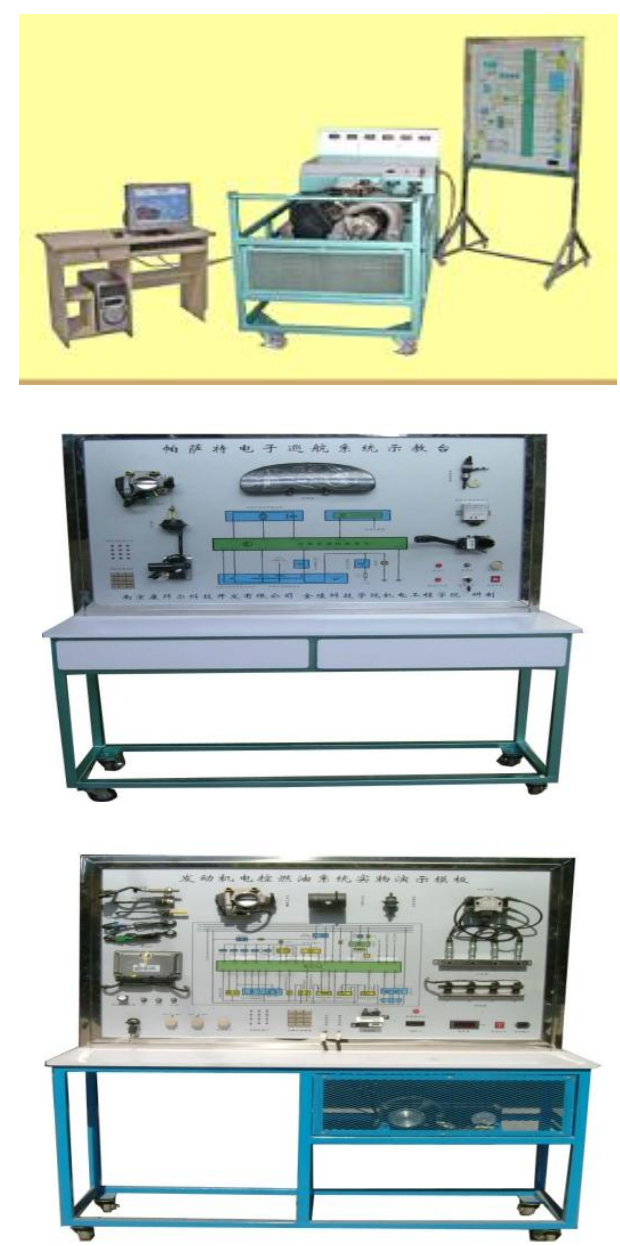

Figure 1 Experimental equipment

2) Fuel System: The students need to point out the installation location of fuel injection nozzle, oil pump, oil pump relay, identify the control circuit diagram of the corresponding drawing, observe the number of each wire harness and it's thickness, color, etc. In addition, students also need to recognize the circuit of each sensor actuator in the schematic diagram of the control system, and bear the connection relationship between them and ECU in their mind. 
3) Ignition system: The students need to point out the installation location of ignition, Holzer sensor, speed sensor, vibration sensor, identify the control circuit diagram of the corresponding drawing, observe the number of each wire harness number and it's thickness, color, etc.. Besides, students also need to distinguish the circuit of each sensor actuator in the schematic diagram of the control system, and learn the connection relationship between them and ECU by heart.

The students also need to master the type of electronic control engine, mainly in the following aspects:

A) To understand the role, installation position, signal characteristics, detection object of air flow meter, and to compare with the intake manifold pressure sensor, and to understand the different advantages and disadvantages of D type and L type.

B) To know the installation position and circuit characteristics of the nozzle, differentiate the different circuit characteristics, advantages and disadvantages of multi-point injection engine and single point injection engine.

C)To master the role of oxygen sensor, it's installation location, signal characteristics, feedback control methods, the comparison between the open loop and closed loop physical differences and conceptual differences, advantages and disadvantages, etc..

D) To understand the function and structure of DIS system (without distributor ignition system), and compare the function, structure principle, advantages and disadvantages of the distributor ignition system.

The automobile cruise control system teaching template can let students understand the connection mode of a cruise control system including sensors, actuators and ECU, a kind of main sensors and actuators and also allow to set fault and check fault diagnosis.

\section{USING MALTAB TO CARRY OUT SIMULATION EXPERIMENT}

This experimental course is oriented to undergraduate students in major of automation (Automotive Electronics Engineering), therefore, the controller's design is much more important for them. So in the lecture, teacher will also address the basic knowledge for design and cultivate their design ability. In addition, students will learn to verify the stability of the controller design with the required needs. MATAB is usually used to verify the simulation.

In the MATAB Simulink, from the automatic transmission control model (shown as Figure 2), in the chart, it can be seen, that the control model of automatic transmission include of an engine model subsystem, a shift logic model sub system, a threshold calculation sub model, a gear sub model and the manipulation graphical interface and output module. According to the vehicle speed shift, as long as open the gear shift logical model (shown as Figure 3), and click the simulation button, then students can observe the whole process of gear up and down. Engine speed and speed can be displayed (shown as Figure 4 and Figure 5).

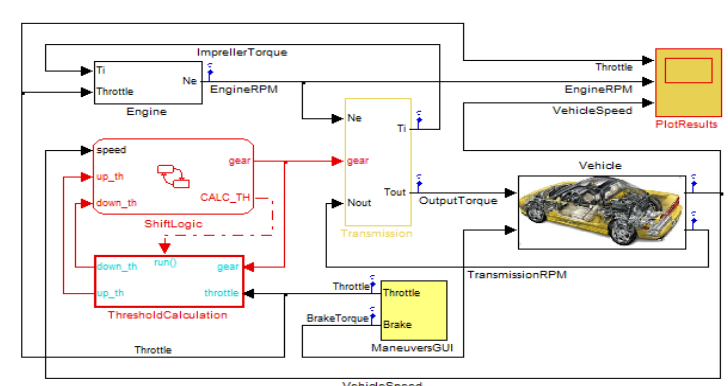

Double-click on ManeuvresGUI and select a maneuver

Figure 2 Automatic transmission control model

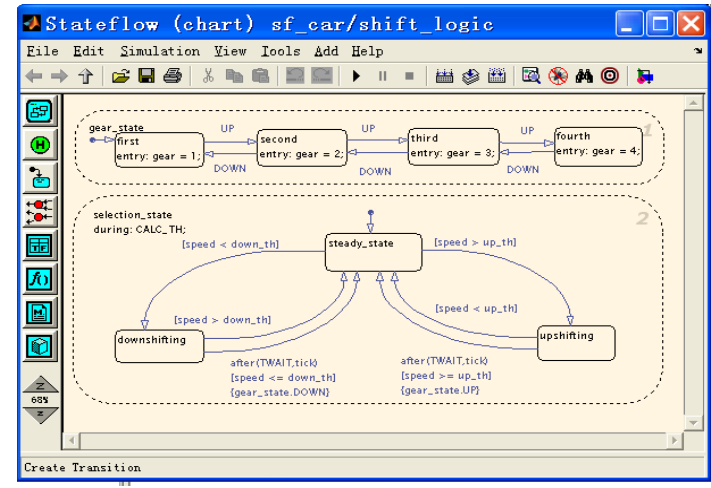

Figure 3 Shift logic

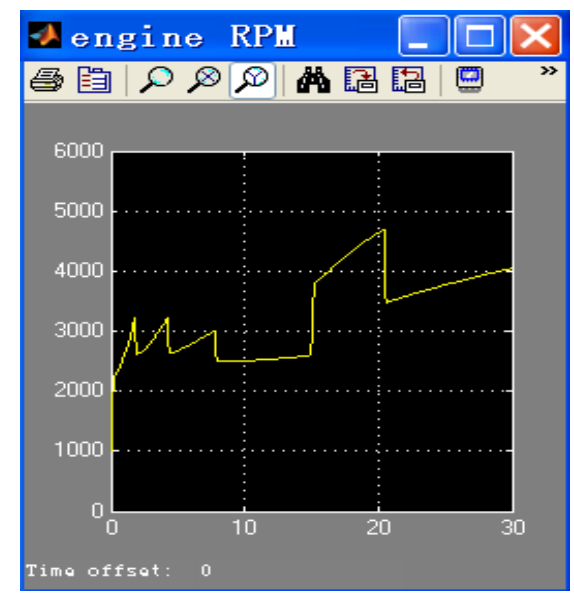

Figure 4 Engine speed

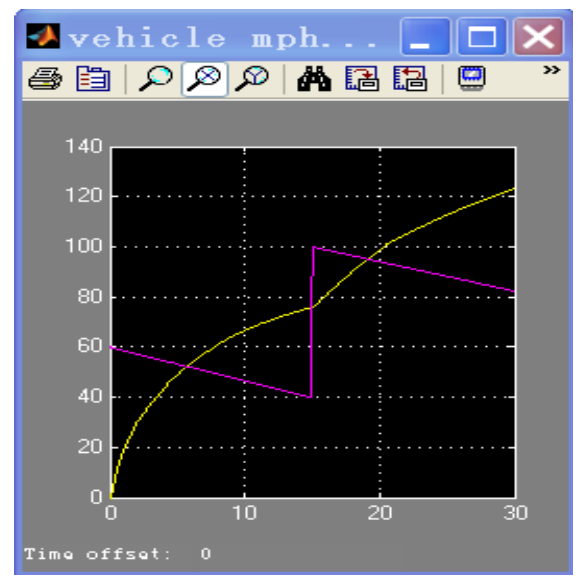

Figure 5 throttle opening and vehicle speed 
In addition to automatic transmission, anti lock braking control system (ABS), suspension control system also allows students to use Matlab simulation. When doing the experiment, we first describe the composition and working principle of the control system, demonstrate the simulation, and then let students to change some of the parameters of the simulation experiment so as to enable students to understand the process of controller designing, and to master the use of Matlab.

\section{DESIGN EXPERIMENTS OF NODE OF CAN BUS BASED ON 51 SINGLE CHIP MICROCOMPUTER}

In the practice of theoretical course and the previous experiment, students have a certain degree of understanding of the composition, working principle and design of the controller, but the actual ability is not enough. So, we consider adding a $51 \mathrm{MCU}$ CAN bus node design experiment.

CAN bus, a serial data communication protocol, is the German Bosch Company from the early 1980s to solve data exchange between controller and measuring instrument in modern cars. It is a kind of multi master bus, communication medium can be twisted pair, coaxial cable and optical fiber. Communication rate can up to $1 \mathrm{Mbps}$. This is the most widely used bus in the car.

In this experiment, teacher will first demonstrate 51 microcontroller programming and explain CAN Bus Principle as an example and then let students try Keli51 programming and debugging, and finally burned the program to write on the mcu. Through this experiment, students can deepen the understanding of the car CAN bus, increasing their interest in the design of the graduation project and will pursue to study hard in this area.

Through this experimental arrangement, 3 weeks of experiment duration let students understanding of the engine, chassis and body control the composition, structure and principle of each subsystem in more detail, also let students have a preliminary understanding of MATLAB simulation and single chip processor usage for design of CAN bus node, let the students to consolidate the theoretical knowledge, lay the foundation for future study and work.

These ideas still need to be improved and perfected in practice, in order to complete the task of experimental teaching.

\section{REFERENCES}

[1] Zhou ChangGen, et al. Construction principle and fault diagnosis of modern automotive electronic control system (Part one): engine part (Fourth Edition) . Beijing Institute of Technology press, 2011.6

[2] Jiang WeiDong, Tian LinLin.Automobile chassis electronic control technology. Machinery Industry Press, 2010.9

[3] Zhang Lei.Principle and maintenance of electronic control system of automobile chassis, Machinery Industry Press, 2012.7
[4] Zhou YunShan. Automotive electronic control technology. Machinery Industry Press, 2004.7

[5] Li JIanQiu, et al. Automotive electronics course. Tsinghua University Press, 2011.5

[6] Luo Xiao, Zhao XingYu,Wu JianZhen et al.Intelligent vehicle lamp control system based on CAN bus, Journal of Shanghai University of Engineering Science, 2015.1

[7] Feng Congyi. Automotive electronic control technology, China Communication Press,2011 